\title{
ROLES OF APOPTOSIS AND AUTOPHAGY ON THE TEXTURE OF RED SEA BREAM MUSCLE
}

\author{
Atsuko Harigaya 1), Reiko Nagasaka ${ }^{2 *}$, Toshiaki Ohshima ${ }^{2)}$ \\ 1)Graduate School of Marine Science and Technology, 4-5-7 Konan, \\ Minato-ku, Tokyo 108-8477, Japan \\ 2)Department of Food Science and Technology, Japan \\ "le-mail : rnagas0@kaiyodai.ac.jp
}

\begin{abstract}
One of commercial brands in farmed-products of red sea bream (Pagrus major), named "Date-Madai", is known to possess hard texture and transparency when served as "Sashimi". The quality of "Sashimi" is usually evaluated by texture, appearance, and color of dark muscle. These characters easily change worse during post mortem storage. The aim of this study was to reveal relationship between proteolytic degradation and muscle quality of the red sea bream. Sensory analysis was carried out to evaluate the quality of "Sashimi" in terms of texture and appearance of flesh. Western blot analysis was conducted to evaluate protein expressions of red sea bream muscle. Significantly higher score in the sensory analysis for brightness was given to the "Date-Madai". Similar tendency were also observed in color, texture and general acceptability. Intracellular effector of the apoptotic pathway includes contributin of caspase family. Lower level in caspase-3 protein was observed in the "DateMadai" muscle. Autophagy is known to be inhibited by the target of rapamycin (TOR) signaling. Phosphorylated ribosomal protein S6 kinase, which is in the downstream of the TOR, increased in the "Date-Madai" muscle. This study concluded that the apoptosis and autophagy could be associated with the quality of the red sea bream.
\end{abstract}

Keywords: apoptosis, autophagy, proteases, red sea bream

\section{INTRODUCTION}

Fish muscle texture is one of the most important quality parameters in considering fish as food, compared to domestic animals' meats. It is well accepted that endogenous proteases in muscle might be appropriate indicators of texture-associated degradation.

The quality of "Sashimi", sliced fresh fish flesh of red sea bream (Pagrus major) is usually evaluated by texture, appearance, and color of dark muscle. These characters easily change worse during transportation and storage at lower temperature. Degradation of the intracellular myofibrillar proteins is known to contribute to muscle softening, namely soft texture (Sato 1999). Apoptosis is a termination mechanism built-in living cells and is induced by certain protein inducers and stress stimuli (Ishida et al. 2003). Caspase-3 induces apoptosis mechanisms in fish muscle (Yabu et al. 2001).

Because the activity of caspase- 3 is induced in fish muscle postmortem, the muscle softening is inhibited by certain caspase inhibitors. Autophagy also contributes to softening of the muscle (Yamashita et al. 1991; Yamashita et al. 2008). The aim of this study was to reveal the relationship between proteolytic degradation and muscle softening of red sea bream. 


\section{MATERIALS AND METHODS}

\section{Sample}

"Date-Madai", one of the farmed red sea bream brands; in Japanese markets was used as sample specimen. The "Sashimi" of "Date-Madai" is known to possess harder texture and transparency. The red sea bream specimens used in this research included the "Date-Madai" and normal farmed red sea bream.

\section{Sensory analysis}

Quality of "Sashimi" was evaluated by sensory analysis. Fourteen panels, who were previously trained and selected by triangle difference tests, compared "Date-Madai" and normal farmed red sea bream in terms of appearance, flavor, color and texture using dosal muscle of fish.

\section{Western blot analysis}

Sodium dodecyl sulphate-polyacrylamide gel electrophoresis (SDS-PAGE) was performed on $10 \%$ separating gel (Laemmli 1970). Caspase-3 and phosphorylated ribosomal protein S6 kinase (p70 S6K) protein expression were evaluated by Western blot analysis. After electrophoresis, the separated molecules were immediately transferred to polyvinylidene difluoride (PVDF) membranes, and the membranes were scanned and the intensities of Western blotting bands were quantified.

\section{RESULTS AND DISCUSSIONS}

\section{Sensory analysis}

Higher score was observed in the texture of the "Sashimi" of "Date-Madai" than that of the normal red sea bream (Figure 1). Significantly higher score for brightness was given to the "Sashimi" of the "Date-Madai" (t-test, ${ }^{*}<0.05$, Figure 2). "Sashimi" of the "Date-Madai" tended to be sperior in color, texture and general acceptability.

\section{Protein expression}

In general, higher autolytic activity of endogenous proteases in major muscle, which is caused by apoptosis and autophagy, induces the hydrolysis of fish muscle proteins. These phenomena contribute to softening of the fish muscle structure during post mortem storage. Upstream signals of the apoptotic pathway induce a caspase family (Szegezdi et al. 2006). In this study, lower caspase-3 protein level was observed in the "Date-Madai" muscle compared to the normal farmed red sea bream muscle (t-test, $*_{p}<0.05$, Table 1).

Autophagy is known to be inhibited by target of rapamycin (TOR) signaling (Noda et al. 1998). The results of the western blot analysis showed that phosphorylated p70 S6K, which locates in the downstream of TOR, tended to increase in the "Date-Madai" muscle compared to the normal farmed red sea bream muscle (Table 1). It has been reported that activated p70 S6K suppresses autophagy (Yamashita et al. 2008). 


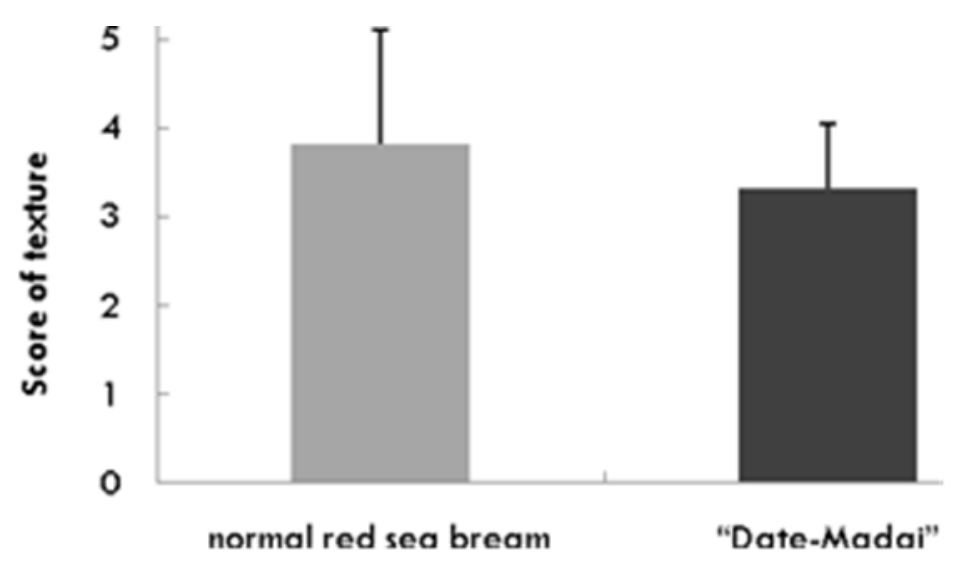

Figure 1. Texture of the red sea bream "Sashimi"

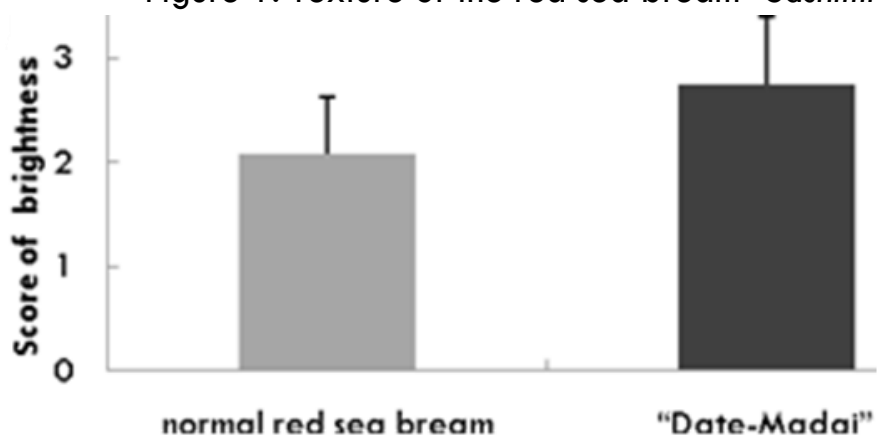

Figure 2. Brightness of the red sea bream "Sashimi" $(* p<0.05)$

Table 1. Protein expression in the red sea bream muscle $\left(n=6, *_{p}<0.05\right)$

\begin{tabular}{ccc}
\hline & Normal red sea bream & "Date-Madai" \\
\hline Caspase-3 & $1.00 \pm 0.116$ & $0.912 \pm 0.0814^{*}$ \\
p70 S6K & $1.00 \pm 0.260$ & $1.22 \pm 0.449$ \\
\hline
\end{tabular}

\section{CONCLUSIONS}

The texture and brightness of the "Sashimi" were superior in the "Date-Madai" to that of normal farmed red sea bream. In the "Date-Madai" muscle, the protein expressions of caspase-3 were lower and that of p70 S6K were higher compared to these of the normal farmed red sea bream muscle. These results clearly suggested that apoptosis and autophagy were inhidited in the "Date-Madai" muscle. Additionally, these results on protein expressions were well coincident with the results of sensory analysis.

This study can conclud that the apoptosis and autophagy could be one of the key factors on the affecting the quality of the skeletal muscle of the red sea bream.

\section{Acknowledgements}

This work was partially supported by JSPS KAKENHI, Grant-in-Aid for Young Scientists (B) and Grant-in-Aid for Scientific Research (A). 


\section{REFERENCES}

Ishida N, Yamashita M, Koizumi N, Terayama M, Ineno T, Minami T. 2003. Inhibition of postmortem muscle softening following in situ perfusion of protease inhibitors in tilapia. Fish. Sci. 69: 632-638.

Laemmli UK. 1970. Cleavage of structural proteins during the assembly of the head of bacteriophage T4. Nature. 227: 680-685.

Mizushima N, Noda T, Ohsumi Y. 1999. Apg 16p is required for the function of the Apg 12pApg5p conjugate in the yeast autophagy pathway. The EMBO J. 18: 3888-3896.

Noda T, Ohsumi Y. 1998. Tor, a phosphatidylinositol kinase homologue controls autophagy in yeast. J.Biol.Chem. 273: 3963-3966.

Sato K, Sakaguchi M, Bremner HA. 1999 Gaping in fish flesh, Extracellular extracellular matrix of fish and shellfish. Research Signpost. Trivandrum. India: 81-94.

Szegezdi E, Logue SE, Samali A. 2006. Mediators of endoplasmic reticulum stress-induced apoptosis. EMBO rep. 7: 880-885.

Yabu T, Yamashita M, Todoriki S. 2001. Stress-induced apoptosis by heat shock, UV and $\gamma$-ray irradiation in zebrafish embryos detected by increased caspase activity and wholemount TUNEL staining. Fish. Sci. 667 (2) : 333-340.

Yamashita $M$, Konagaya S. 1991. Hydrolytic action of salmon cathepsins $B$ and $L$ to muscle structural proteins in respect of muscle softening. Nippon Suisan Gakkaishi. 57: 19171922.

Yamashita M, Yabu T. 2008. Observation of stress-induced autophagy in fish cells. Bull. Fish. Res. Agen. 26: 23-28. 